\title{
Properties of simulated Milky Way-mass galaxies in loose group and field environments ${ }^{\star}$
}

\author{
C. G. Few ${ }^{1}$, B. K. Gibson ${ }^{1,2,3}$, S. Courty ${ }^{4}$, L. Michel-Dansac ${ }^{4}$, C. B. Brook ${ }^{5}$, and G. S. Stinson ${ }^{6}$ \\ 1 Jeremiah Horrocks Institute, University of Central Lancashire, Preston, PR1 2HE, UK \\ e-mail: cgfew@uclan.ac.uk \\ 2 Department of Astronomy \& Physics, Saint Mary's University, Halifax, Nova Scotia, B3H 3C3, Canada \\ 3 Monash Centre for Astrophysics, Monash University, 3800 Victoria, Australia \\ ${ }^{4}$ Université de Lyon, Université Lyon 1, Observatoire de Lyon, CNRS, UMR 5574, Centre de Recherche Astrophysique de Lyon, \\ École Normale Supérieure de Lyon, 9 avenue Charles André, 69230 Saint-Genis Laval, France \\ 5 Grupo de Astrofísica, Departamento de Fisica Teorica, Modulo C-15, Universidad Autónoma de Madrid, 28049 Cantoblanco, Spain \\ ${ }^{6}$ Max-Planck-Institut für Astronomie, Königstuhl 17, 69117 Heidelberg, Germany
}

Received 22 May 2012 / Accepted 27 September 2012

\section{ABSTRACT}

\begin{abstract}
Aims. We test the validity of comparing simulated field disk galaxies with the empirical properties of systems situated within environments more comparable to loose groups, including the Milky Way's Local Group.

Methods. Cosmological simulations of Milky Way-mass galaxies have been realised in two different environment samples: in the field and in loose groups environments with similar properties to the Local Group. Apart from the differing environments of the galaxies, the samples are kept as homogeneous as possible with equivalent ranges in last major merger time, halo mass and halo spin. Comparison of these two samples allow for systematic differences in the simulations to be identified. A kinematic decomposition is employed to objectively quantify the spheroid-to-disk ratio and to isolate the disk-star population. Metallicity gradients, disk scale lengths, colours, magnitudes and age-velocity dispersion relations are studied for each galaxy in the suite and the strength of the link between these and environment of the galaxies is studied.

Results. Metallicity gradients are consistent with observations of HII regions in spiral galaxies and, in agreement with observations, correlate with total galaxy mass. The bulge-to-disk ratio of the galaxies show that these galaxies are less spheroid dominated than many other simulated galaxies in literature with the majority of both samples being disk dominated. We find that secular evolution and mergers dominate the spread of morphologies and metallicity gradients with no visible differences between the two environment samples. In contrast with this consistency in the two samples there is tentative evidence for a systematic difference in the velocity dispersion-age relations of galaxies in the different environments. Loose group galaxies appear to have more discrete steps in their velocity dispersion-age relations, if this is true it suggests that impulsive heating is more efficient in the stars of galaxies in denser environment than in the field. We conclude that at the current resolution of cosmological galaxy simulations field environment galaxies are sufficiently similar to those in loose groups to be acceptable proxies for comparison with the Milky Way provided that a similar assembly history is considered.
\end{abstract}

Key words. Local Group - Galaxy: formation - galaxies: evolution - methods: numerical

\section{Introduction}

It is well established that galaxy interactions and mergers result in significant changes in a system's star formation rate (Barton et al. 2000; Lambas et al. 2003; Nikolic et al. 2004; Ellison et al. 2008) and its chemical properties (Donzelli \& Pastoriza 2000; Márquez et al. 2002; Fabbiano et al. 2004; Kewley et al. 2006; Michel-Dansac et al. 2008; Rupke et al. 2008; Kewley et al. 2010; Sol Alonso et al. 2010). Studies have shown that in denser large scale environments the star formation rate of galaxies tends to be lower (Gómez et al. 2003). It is often suggested that galaxies in clusters are older and have therefore consumed the limited gas available for star formation (Lilly et al. 1996), or that proximity to other galaxies means that the reservoir of infalling gas must be shared (Lewis et al. 2002). It is also thought that a dominant method of reducing the star formation rate in dense environment is through ram pressure stripping that removes the gas envelope from field galaxies as they are accreted to groups and clusters (Balogh et al. 2004b).

* Appendix A is available in electronic form at http://www . aanda.org
Proximity to a cluster centre is also known to impact the morphology of galaxies and clusters have a greater fraction of early type galaxies; i.e. the so-called morphology-density relation (Dressler et al. 1997). While the accretion of field galaxies into denser environments may strip gas from the galaxy and leave a slowly reddening S0 galaxy, it will do little else to alter the morphology. Morphological transformations are therefore attributed to gravitational interactions with other group members (Moore et al. 1996; Weinmann et al. 2006). Denser environments also increase the likelihood of galaxy mergers. This is supported by the findings of McGee et al. (2008) where an enhancement in the number of asymmetric disks is found in group environments (these groups typically have velocity dispersions less than $700 \mathrm{~km} \mathrm{~s}^{-1}$, smaller than the larger clusters considered in many other works). The authors also put forward the intriguing conclusion that while the groups exhibit a larger fraction of galaxies that are bulge dominated, no evidence is found that the group environment has any effect on the bulk properties of the disk galaxy population.

Mergers have a direct impact on both the star formation history of the galaxies and the metal distribution. Hydrodynamical 
simulations by Hernquist (1989) and Barnes \& Hernquist (1996) show that mergers funnel gas into the central regions of galaxies. This would tend to dilute the gas phase metallicity at small radii and trigger centrally concentrated star formation (Rupke et al. 2010; Montuori et al. 2010; Perez et al. 2011). This trend is consistent with observations of flattened metallicity gradients in luminous and ultraluminous infrared galaxies that are identified as merged systems (Rupke et al. 2008) and in interacting galaxy pairs (Ellison et al. 2008; Michel-Dansac et al. 2008; Kewley et al. 2010) where the influence on star formation rates extends to projected separations of up to $40 h^{-1} \mathrm{kpc}$ (Ellison et al. 2008).

Interactions are far more common in denser environments and one would expect to see the effect of these interactions imprinted on the metallicity of cluster and group galaxies when compared with those in the field. Cooper et al. (2008) observe that not only do members of clusters have greater metallicities but also that on average galaxies that are closer to other cluster members have greater oxygen abundances (an effect of order $0.05 \mathrm{dex}$ ). The conclusion of the work is that metallicity effects are not driven by the cluster as a whole but only by the specific proximity of each galaxy to others; consistent with past findings (Balogh et al. 2004a; Martínez et al. 2008). The independence of metallicity on large-scale environment is perhaps refuted by the findings of Ellison et al. (2009) where a residual metallicity-environment effect is found observationally even after the dependence on luminosity and colour have been accounted for, however these two results are not entirely irreconcilable. Martinez-Vaquero et al. (2009) select simulated dark matter systems based on the mass and circular velocity of haloes, the mutual proximity of the haloes and the distance to a halo with the same mass as the Virgo cluster. These simulations explore the properties of the haloes while relaxing these criteria and find that the nearness of massive external haloes is the most significant factor determining the dispersion of Local Group systems: the coldness of the Local Group can be attributed mostly to its isolation from clusters.

Recent simulations have employed higher resolution and more advanced supernova feedback prescriptions which ameliorate the traditional failings of galaxy simulations (Robertson et al. 2004; Governato et al. 2007; Scannapieco et al. 2009; Sánchez-Blázquez et al. 2009; Stinson et al. 2010; Rahimi et al. 2010; Brooks et al. 2011). It is now possible (and prudent) to examine the more subtle factors that influence galaxy properties. Given the demonstrable difference between galaxies in different (albeit extremely so) environments it is reasonable to expect that galaxies in loose groups such as our own Local Group may differ from those in field.

At present the majority of literature on this topic focusses on constrained simulations based upon cosmological initial conditions that will purposefully give rise to systems with the properties of the Local Group (Gottloeber et al. 2010; Peirani 2010; Libeskind et al. 2011; Peirani et al. 2012) or the use of extremely large simulation volumes to search for analogous systems (Snaith et al. 2011, and references therein). Here we provide a complementary approach by identifying Local Group analogues in a suite of hydrodynamical simulations. In what follows, we will explore the hypothesis that simulated isolated field galaxies can be considered suitable proxies for Local Group analogues.

The purpose of this work is not to reproduce artificial clones of the Local Group but rather to gauge the systematic offset in properties between field galaxies and those with a similar degree of interaction with neighbours such as is encountered between the Milky Way and Andromeda. These groups are henceforth termed as "loose groups" to be clear that the systems in the loose group sample are not Local Group clones from initial conditions designed to reproduce the exact layout of the local universe but are chosen from cosmological simulations based on certain similarities to the Local Group. In doing so we find galaxies with a range of merger histories that nonetheless produce disk galaxies. It is hoped that the discrepancy between the properties of these two samples will place constraints on future simulations and present insight into the failings of field galaxy simulations when attempting to recover the properties of Local Group galaxies. The method employed in producing this sample and the properties of the simulation code are described in Sect. 2. In Sect. 3 we present an analysis of the galaxy disk fractions, colours, magnitudes, metallicity gradients and velocity dispersions and discuss these results in Sect. 4.

\section{Method}

\subsection{Simulations}

The galaxies presented here were simulated using the adaptive mesh refinement code RAMSES (v3.01; Teyssier 2002). RAMSES is a three-dimensional Eulerian hydrodynamical code with an $N$-body particle-mesh scheme to compute self-gravity. The mesh automatically refines according to the local particle density in addition to a static refinement of nested regions that reduces the run-time while maintaining high resolution around the galaxy of interest. Details of the refinement scheme are described by Teyssier (2002). RAMSES includes density- and metallicitydependent radiative cooling rates, using an ionisation equilibrium with an ultra-violet radiative background (Haardt \& Madau 1996). Gas cells with a density $\rho$ exceeding a star formation threshold of $\rho_{0}=0.1 \mathrm{~cm}^{-3}$ form stars at a rate of $\dot{\rho}=-\rho / t_{\star}$. The star formation timescale $t_{\star}$ is itself a function of the density and the free-fall time through the free parameter, $t_{0}$, as follows: $t_{\star}=$ $t_{0}\left(\rho / \rho_{0}\right)^{-1 / 2}$ (Dubois \& Teyssier 2008). We use $t_{0}=8 \mathrm{Gyr}$, corresponding to a $2 \%$ star formation efficiency for $\rho_{0}=0.1 \mathrm{~cm}^{-3}$. We use the kinetic feedback mode of RAMSES that aims to reproduce the energetic and chemical enrichment of SNeII explosions: after $10^{7}$ years, star particles release some mass, momentum and energy into a 2-cell radius feedback-sphere centred on the star particle. We characterise the initial mass fraction of the star particles with the parameter $\eta_{\mathrm{SN}}=10 \%$ (which corresponds to the mass fraction of stars contributing supernovae feedback) and we do not use any mass loading factor. The energy injected into the gas phase is in the form of kinetic energy with $100 \%$ efficiency (i.e. $10^{51} \mathrm{erg} \mathrm{SN}^{-1}$ ). Chemical enrichment of the gas is followed through the global metallicity $Z$ using a yield of $10 \%$. RAMSES includes a polytropic equation of state with an index of $5 / 3$ to prevent non-physical gas fragmentation in all gas cells with an hydrogen density larger than the star formation density threshold. The temperature threshold is $T_{\text {th }}=10^{4} \mathrm{~K}$ and in the analysis and Table 2 in particular, we will set the temperature of the high-density gas cells to $10^{4} \mathrm{~K}$ to account for unresolved cold gas. A detailed description of the star formation and feedback treatments may be found in Dubois \& Teyssier (2008), though the v3.01 RAMSES has a different technical implementation of the kinetic feedback.

Candidate haloes for this work were identified from dark matter simulations and then resimulated with baryonic physics and a more refined grid around regions of interest, using the same technique as in Sánchez-Blázquez et al. (2009). The simulations are conducted in a cosmological framework with $H_{0}=$ $70 \mathrm{~km} \mathrm{~s}^{-1} \mathrm{Mpc}^{-1}, \Omega_{\mathrm{m}}=0.28, \Omega_{\Lambda}=0.72, \Omega_{\mathrm{b}}=0.045$, and 
$\sigma_{8}=0.8$. Two volumes are used with a size of $20 h^{-1} \mathrm{Mpc}$ and $24 h^{-1} \mathrm{Mpc}$ and the maximum refinement achieved (16 levels) corresponds to $436 \mathrm{pc}$ and $523 \mathrm{pc}$ respectively. The dark matter mass resolution is $5.5 \times 10^{6} M_{\odot}$ and $9.5 \times 10^{6} M_{\odot}$ respectively. We now describe the selection of our candidate haloes.

\subsection{Environment}

A rich literature exists comparing simulated field disk galaxies with observations of the Milky Way (e.g. Brook et al. 2004; Scannapieco et al. 2005; Sánchez-Blázquez et al. 2009; Kobayashi \& Nakasato 2011; House et al. 2011; Guedes et al. 2011). The purpose of our work here is to determine whether or not environments comparable to those of the Local Group the true environment of the Milky Way-result in any measurable characteristics which would call into question this fundamental tenet of simulation vs. observation comparison.

The dark matter haloes of the galaxies presented here are chosen as follows. Dark matter haloes with virial mass $\left(M_{\mathrm{vir}}\right)$ in the range $5 \times 10^{11}-10^{12} M_{\odot}$ are considered as loose group candidate haloes. Further selection criteria for the loose group sample are such that large groups are excluded; each halo must have at least one companion with a comparable virial mass at a distance of 500-700 kpc but have no haloes more massive than $5 \times 10^{12} M_{\odot}$ within $5 \mathrm{Mpc}$. Please note that the loose groups are not specifically constrained to be pairs, rather they may include up to four haloes each with $M_{\text {vir }}>10^{11} M_{\odot}$.

The field sample consists of galaxies that have no other dark matter haloes of mass $M_{\text {vir }}>3 \times 10^{11} M_{\odot}$ within a $3 \mathrm{Mpc}$ radius. These field environment galaxies are far more common than the loose groups and those presented here were chosen based upon properties such as mass, spin factor and number of mergers such that a similar range in each of these can be found in both the loose group and field samples. The spin factors of the dark matter haloes range from 0.007 to 0.08 , this range encompasses the majority of dark matter halos and is simply used to exclude outliers. The merger trees of the galaxies are used to select loose group and isolated haloes to avoid galaxies (in both loose group and field samples) that have any significant mergers after $z=1$. This is done to ensure that disk galaxies are formed and also prevents the increased likelihood of mergers in denser environments from dominating the systematic differences. The full sample of galaxies covers a range in virial mass from $10^{11}-10^{12} M_{\odot}$ and includes the field galaxies and all the group galaxies in this mass range, i.e. massive satellites in addition to the dominant group members.

There are ten loose group galaxies (taken from three groups) and nine field galaxies. The dark matter distributions of a field galaxy and a loose group are shown in Fig. 1. Each sample is roughly divided into two different resolutions corresponding to the different cosmological volumes. None of the galaxies have passed pericenter with one another as of $z=0$ and so one should not expect dramatic tidal or merger effects. Two of the galaxies do have recent mergers, but for the analysis presented here are studied at an earlier timestep (that is unaffected by the $z=0$ merger, as described later). The details of each galaxy can be found in Table 1. It is well known that the stellar mass fraction of simulated galaxies is too high, particularly for older stellar populations. This is true of the galaxies presented here (stellar masses are stated in Table 1) with the stellar-to-total mass fraction a factor of 2-3 times too high when counting all mass in the virial radius (Mandelbaum et al. 2006; Moster et al. 2010; Leauthaud et al. 2012). We do not believe that this has a drastic impact on this analysis. Firstly, the issue affects galaxies independent

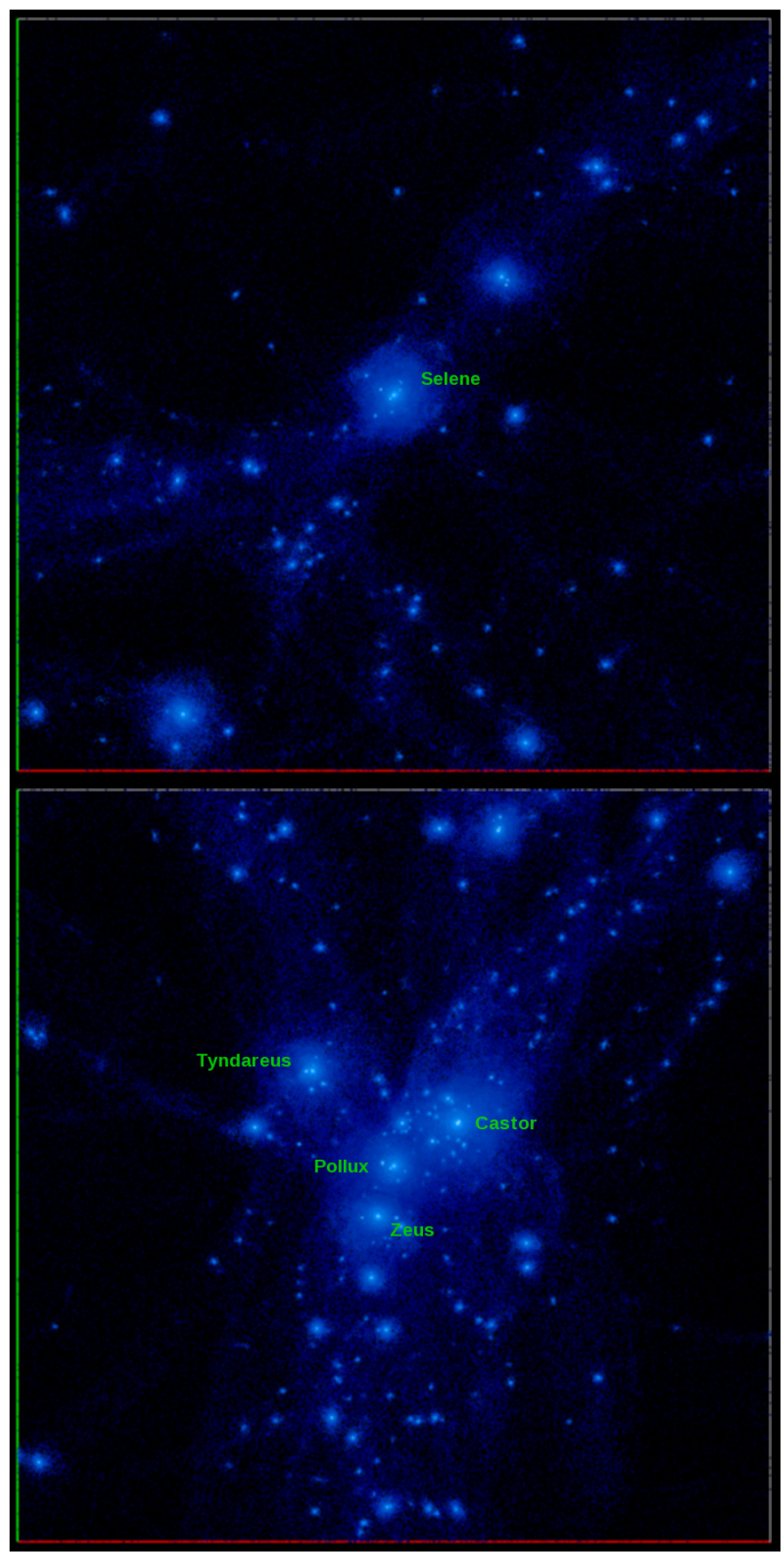

Fig. 1. Examples of the different environments considered in this work. The dark matter haloes of the field galaxy Selene (top) and the loose group galaxies Castor, Pollux, Tyndareus and Zeus (bottom) are shown as blue particles (lighter colours correspond to denser regions). Images are $4 \times 4 \mathrm{Mpc}^{2}$ in size and have a depth of $4 \mathrm{Mpc}$.

of environment, so comparisons of the loose group and field samples are not systematically offset by this effect. Secondly, the early formation of stars would lead to an overly massive spheroid. We will show later that the spheroid mass is not extreme, although admittedly the disk stars are too concentrated, i.e. disk scale lengths are too short (see Sect. 3.2).

A preliminary study of the metallicity gradients in these galaxies has been conducted in comparison with metallicity gradients found in other simulated galaxies and in semi-numerical models in Pilkington et al. (2012) (there these galaxies are 
A\&A 547, A63 (2012)

Table 1. List of the disk galaxies simulated for this work, with their total, dark matter, baryonic, stellar and gaseous masses.

\begin{tabular}{|c|c|c|c|c|c|c|c|}
\hline Name & Environment & $\begin{array}{l}\text { Resolution } \\
\text { (pc) }\end{array}$ & $\begin{array}{l}M_{\text {tot }} \\
\left(M_{\odot}\right)\end{array}$ & $\begin{array}{l}M_{\mathrm{DM}} \\
\left(M_{\odot}\right)\end{array}$ & $\begin{array}{c}M_{\text {baryon }} \\
\left(M_{\odot}\right)\end{array}$ & $\begin{array}{c}M_{\text {stellar }} \\
\left(M_{\odot}\right)\end{array}$ & $\begin{array}{l}M_{\text {gas }} \\
\left(M_{\odot}\right)\end{array}$ \\
\hline Castor & loose group & 436. & $1.05 \times 10^{12}$ & $8.70 \times 10^{11}$ & $1.77 \times 10^{11}$ & $1.10 \times 10^{11}$ & $6.76 \times 10^{10}$ \\
\hline Pollux & loose group & 436. & $4.23 \times 10^{11}$ & $3.48 \times 10^{11}$ & $7.54 \times 10^{10}$ & $4.99 \times 10^{10}$ & $2.55 \times 10^{10}$ \\
\hline Zeus & loose group & 436. & $2.33 \times 10^{11}$ & $1.97 \times 10^{11}$ & $3.60 \times 10^{10}$ & $2.57 \times 10^{10}$ & $1.03 \times 10^{10}$ \\
\hline Tyndareus & loose group & 436. & $3.30 \times 10^{11}$ & $2.82 \times 10^{11}$ & $4.82 \times 10^{10}$ & $3.24 \times 10^{10}$ & $1.59 \times 10^{10}$ \\
\hline Apollo & loose group & 523. & $8.94 \times 10^{11}$ & $7.39 \times 10^{11}$ & $1.55 \times 10^{11}$ & $1.06 \times 10^{11}$ & $4.89 \times 10^{10}$ \\
\hline Artemis & loose group & 523. & $7.45 \times 10^{11}$ & $6.46 \times 10^{11}$ & $9.84 \times 10^{10}$ & $5.61 \times 10^{10}$ & $4.23 \times 10^{10}$ \\
\hline Daphne & loose group & 523. & $3.09 \times 10^{11}$ & $2.58 \times 10^{11}$ & $5.14 \times 10^{10}$ & $2.90 \times 10^{10}$ & $2.24 \times 10^{10}$ \\
\hline Leto & loose group & 523. & $2.49 \times 10^{11}$ & $2.05 \times 10^{11}$ & $4.38 \times 10^{10}$ & $2.92 \times 10^{10}$ & $1.46 \times 10^{10}$ \\
\hline Luke & loose group & 523. & $1.13 \times 10^{12}$ & $9.40 \times 10^{11}$ & $1.88 \times 10^{11}$ & $1.07 \times 10^{11}$ & $8.13 \times 10^{10}$ \\
\hline Leia & loose group & 523. & $3.93 \times 10^{11}$ & $3.25 \times 10^{11}$ & $6.76 \times 10^{10}$ & $4.75 \times 10^{10}$ & $2.01 \times 10^{10}$ \\
\hline Ben & field & 523. & $7.74 \times 10^{11}$ & $6.42 \times 10^{11}$ & $1.32 \times 10^{11}$ & $8.16 \times 10^{10}$ & $5.08 \times 10^{10}$ \\
\hline Tethys & field & 523. & $7.21 \times 10^{11}$ & $5.94 \times 10^{11}$ & $1.27 \times 10^{11}$ & $8.21 \times 10^{10}$ & $4.51 \times 10^{10}$ \\
\hline Krios & field & 523. & $5.68 \times 10^{11}$ & $4.78 \times 10^{11}$ & $8.98 \times 10^{10}$ & $6.15 \times 10^{10}$ & $2.84 \times 10^{10}$ \\
\hline Atlas & field & 523. & $6.48 \times 10^{11}$ & $5.55 \times 10^{11}$ & $9.35 \times 10^{10}$ & $6.15 \times 10^{10}$ & $3.20 \times 10^{10}$ \\
\hline Hyperion & field & 523. & $1.03 \times 10^{12}$ & $8.58 \times 10^{11}$ & $1.72 \times 10^{11}$ & $1.13 \times 10^{11}$ & $5.92 \times 10^{10}$ \\
\hline Eos & field & 436. & $4.64 \times 10^{11}$ & $3.92 \times 10^{11}$ & $7.19 \times 10^{10}$ & $4.68 \times 10^{10}$ & $2.52 \times 10^{10}$ \\
\hline Helios & field & 436. & $1.05 \times 10^{12}$ & $8.91 \times 10^{11}$ & $1.62 \times 10^{11}$ & $1.23 \times 10^{11}$ & $3.93 \times 10^{10}$ \\
\hline Selene & field & 436. & $6.07 \times 10^{11}$ & $5.09 \times 10^{11}$ & $9.87 \times 10^{10}$ & $6.71 \times 10^{10}$ & $3.16 \times 10^{10}$ \\
\hline Oceanus & field & 436. & $1.12 \times 10^{12}$ & $9.19 \times 10^{11}$ & $1.97 \times 10^{11}$ & $1.41 \times 10^{11}$ & $5.59 \times 10^{10}$ \\
\hline
\end{tabular}

Notes. The spatial resolution of the simulation they originate from and their host environment is also quoted. The properties are calculated at $z=0$ except for Castor and Eos as described in Sect. 2.3.

referred to as Ramses Disk Environment Study or RaDES galaxies); that work demonstrated that when compared with other models the RaDES galaxies tend to possess shallower metallicity gradients and have a more subtle evolution as a function of time, something the authors attribute to the more uniform star formation profile compared to other models (Pilkington et al. 2012). This issue is discussed further in Sect. 3.2.

\subsection{Merger history}

The predominant strength of using cosmological simulations to develop this suite of galaxies is that a self-consistent merger tree provides the basis of the evolution for each galaxy. This ensures the conditions in the environments selected here are consistent with the current understanding of cosmology and not biased by artificial initial conditions that may affect the results. We begin by demonstrating that there is at least a superficial similarity between the merger histories of the two samples in spite of the environmental differences.

At each timestep a catalogue of haloes and subhaloes is created using the Adaptahop algorithm (Aubert et al. 2004). The halo catalogues are then linked into merger trees for the selected haloes using the "most massive substructure method" detailed in Tweed et al. (2009). Under this formalism, at any given branch in the tree the most massive progenitor is considered the parent halo.

It is not however a trivial exercise to define mergers in this context, and the following definition of a merger is adopted: a merger occurs when an object is identified as a subhalo in a given output but not in the previous output. In practice, the subhalo continues to exist as an identifiable structure orbiting the host halo for several Gyr. Due to dynamical friction, the subhalo gradually sinks into the potential well of the host halo and is slowly stripped of mass before dissolving completely, at which point all particles are attributed to the host halo. We found that in general with the merger time definition above there is a delay of up to $4 \mathrm{Gyr}$ between the merger time and any real interaction with the disk, i.e., there is a delay time between the dark matter merger and the galaxy disk merger. The definition above is used because the amount of mass loss before the subhalo dissolves is unpredictable and the mass ratio of subhalo to host is a more useful quantity for the purpose of evaluating the magnitude of the merger. The magnitude and timing of mergers for each galaxy are shown in Fig. 2. This figure shows that most mergers a galaxy experiences occur at early times and that these early mergers tend to have mass ratios closer to unity. This is partly due to universal expansion reducing the merger likelihood and partly to the limited size of haloes at early times limiting the mass range and making equal mass mergers more likely.

The traditional definition of a major-merger, $M_{\text {host }} / M_{\text {sub }} \leq 3$, has been made significantly more generous $\left(M_{\text {host }} / M_{\text {sub }} \leq 10\right)$ in this work due to the low number of 3:1 mergers in the sample. The low number of such mergers is due to selection effects, these galaxies are chosen from dark matter haloes based partly on the merger trees and haloes with many mergers were discounted as unsuitable for hosting disk galaxies. A commonly used metric of the merger history is the time at which the last major-merger took place. At this point we note that two of the galaxies (identified by the names, Castor and Eos) show obvious signs of disturbance at $z=0$ due to an ongoing merger that makes the disk of the galaxy difficult to identify. The presence of mergers may at first be considered counter to the previously mentioned constraint that the galaxies have no recent major mergers, in both cases the merger did not appear in the dark matter-only simulation and only became apparent following the inclusion of baryons. We remedy this in both cases by analysing the galaxy disk at a timestep immediately preceding the disturbance. While these galaxies appears in the analysis as a low redshift $(z \simeq 0.03)$ late-type object, they may also be considered as more local irregular galaxies if the $z=0$ output is analysed. We conclude this section by stressing that the objective here is not to quantify the effect of mergers on disk galaxies but to examine what effect the environment might have (i.e. via ambient effects) when the increased instance of mergers is discounted. As such we point to 


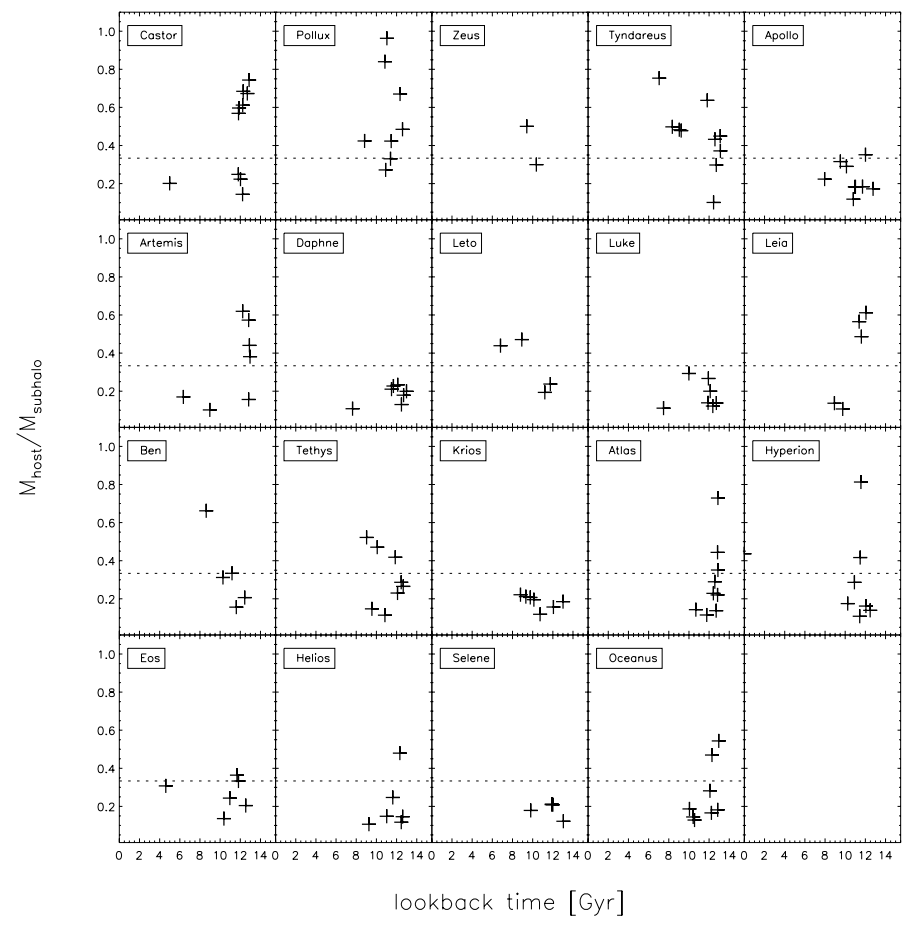

Fig. 2. Major mergers distributions, the top two rows are loose group galaxies and the bottom two rows are field galaxies, a convention that is followed throughout this work. The magnitude of the merger is represented by the virial mass of the host halo divided by the virial mass of the merging body. As stated in the text, mass ratios are calculated at the time the haloes first come into contact as the mass associated with merging structures is often greatly diminished by the time the disk is disrupted. The majority of mergers take place at early times when the galaxy halo still has a low mass and assembly is mostly hierarchical. We find that only mergers shown above to occur further back than $4 \mathrm{Gyr}$ have any significant influence on the disk structure and that mergers can take several Gyr before they have descended far enough into the halo to disrupt the disk. A dashed line shows the lower bound of the commonly used definition of major mergers.

Fig. 2 as evidence that the loose group galaxies have the same diversity of merger history as do the field galaxies.

\subsection{Disk decomposition}

We now separate the stellar particles into spheroidal and disk populations using a kinematic decomposition similar to that of Abadi et al. (2003). Details on the decomposition for each galaxy can be found in the appendix where the orbital circularity distributions are presented. In short, stars are assumed to belong to either a spheroidal or a rotating disk component through analysis of the orbital circularity, i.e. the ratio of their angular momentum, $J_{z}$ to the circular orbit angular momentum $J_{\text {circ }}$ for a given particle energy. The successes of this method are shown in Fig. A.2. It should be noted that while the $J_{z} / J_{\text {circ }}$ distribution has two components, in the intermediate region, particles are stochastically attributed to each component. Thus it is not necessarily true that stars identified as belonging to the spheroid did not form as disk stars. As such the spheroidal component will contain disturbed disk stars (which is arguably appropriate) and more critically, circularly rotating bulge stars will be attributed to the inner regions of the decomposed disk. This does not skew the spheroid-to-disk mass ratio as the vast majority of star particles have equal mass, furthermore the order of this effect should be small as shown in Fig. A.1 which plots the star formation history for the entire galaxy (solid line) and the disk (dashed line) and demonstrates that the selected disk stars well represent the stars formed at late times.

In the analysis that follows we define a disk annulus to exclude contamination by bulge stars and avoid halo stars at the disk edge. Figure 3 shows the rotation curves for the galaxies (and circular velocities for each phase of matter) and choice of disk annulus (indicated by diamond symbols at an arbitrary vertical position). The outer extent of the stellar disk region is first constrained by examination of the rotation curve of the young stars (less than $100 \mathrm{Myr}$ old), the departure of the young stars rotation curve (solid red line) from the gaseous rotation curve (solid blue line) is a useful indicator of the stellar disk edge. Consideration is also given to the stellar density profiles and metallicity gradients of each galaxy, in several cases the density profile or metallicity gradient extends beyond or falls short of the young disk edge. The final disk annulus is conservative to allow for gradients to be measured for each property over a consistent region while avoiding bias from unusual features. Despite this there are cases where the region over which gradients and scale-length are determined has been changed to reflect the characteristics of the galaxy in question. A summary of the galaxy properties can be found in Table 2 and star formation histories for each galaxy are plotted in Fig. A.1.

\subsection{Mock observations}

The galaxy's stellar and gaseous distributions, ages and metallicities have been used by the ray tracing programme SUNRISE (Jonsson 2006) to produce mock images. The Starburst 99 stellar population models (Leitherer et al. 1999) define the colour and magnitude of stellar particles. Scattering and extinction are determined by assuming that dust follows the gas phase metallicity distribution. Mock images of the galaxies may be found in Fig. 4, each image being $50 \times 50 \mathrm{kpc}^{2}$ in size and produced using SDSS $g, r$ and $i$ filters. We draw the reader's attention to the asymmetry of the more extended disks of Luke and Oceanus, the warped disks of Castor, Tyndareus, Krios and Hyperion and to the red, spheroid-dominated Helios.

\section{Results}

\subsection{Disk fraction}

One of the potential differences that may be seen between the galaxies in different environments is the bulge-to-disk ratio. There is a body of evidence suggesting that a morphologydensity relation exists (Dressler 1980; Giuricin et al. 1995; Bamford et al. 2009) due to harassment by neighbours and the increased likelihood of mergers. The spheroid-to-disk stellar mass ratios for the sample are shown in Fig. 5 and it is immediately apparent that the majority are disk dominated (spheroid/total $<1$ ) in spite of the spheroid containing the net mass of the halo in addition to the bulge. Only the smallest group members are spheroid dominated (Leto, Tyndareus and Zeus) with many of the larger galaxies having disk masses exceeding spheroid masses by a factor of 2-4. There is no noticeable tendency for the galaxies with more disturbed disks and smaller galaxies to be spheroid dominated; Artemis has a disturbed disk and yet still exhibits a spheroid-to-disk ratio of 0.326 , likewise the low mass galaxies Daphne, Pollux and Eos have quite strong disk components in contrast with the similarly less massive $\left(M_{\mathrm{vir}}<4 \times 10^{11} M_{\odot}\right)$ galaxies Zeus, Tyndareus and Leto. Given the typical problem of forming too many stars, particularly at 


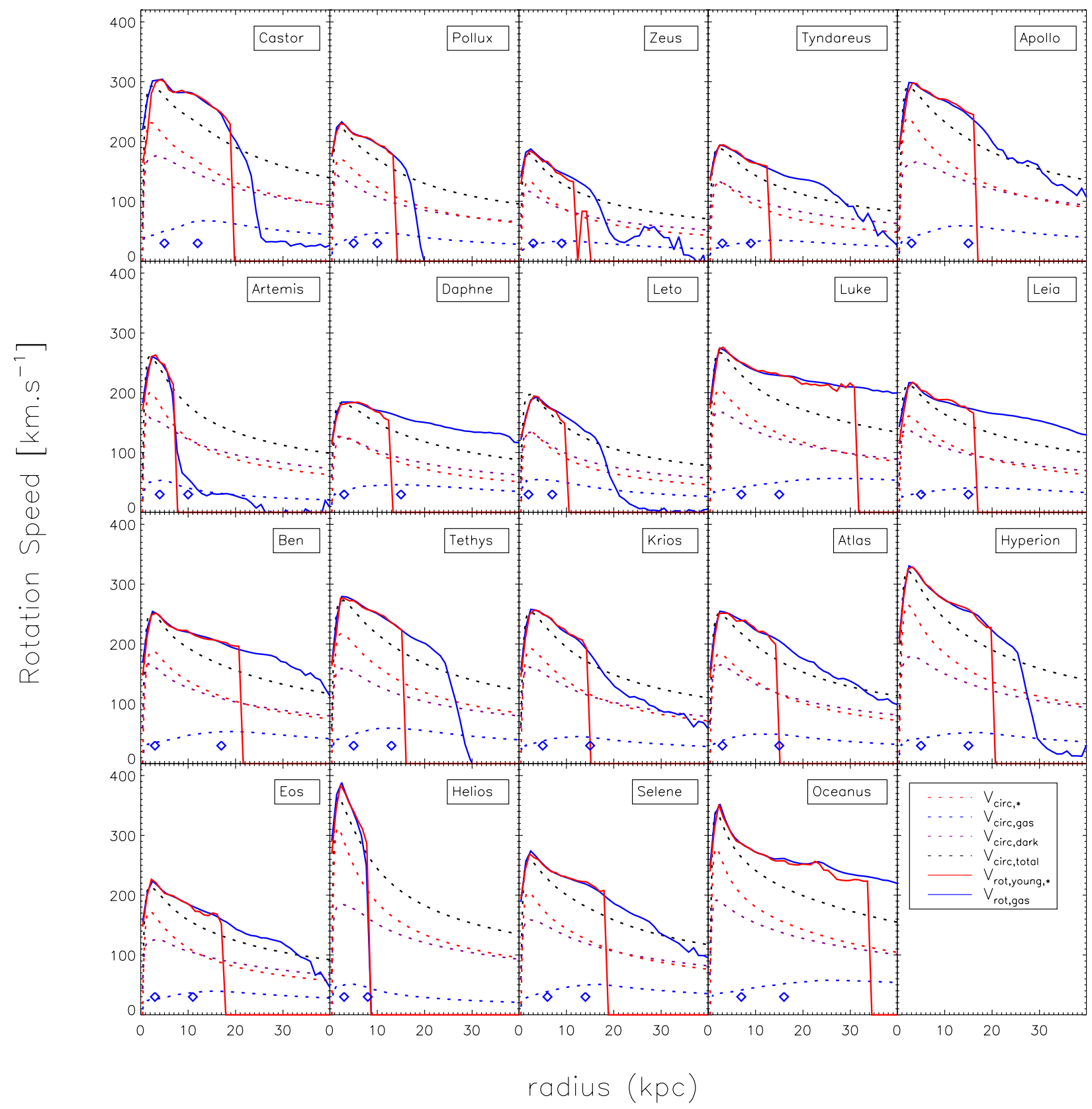

Fig. 3. Rotation curves showing the circular velocity for stars (red), gas (blue) and dark matter (black) as dashed lines and rotation velocities (solid lines) of young stars (age $<100 \mathrm{Myr}$ at $z=0$ ) and gas as a function of radius. Two blue diamonds denote the inner and outer "disk radii" at an arbitrary vertical position. These values are chosen from inspection of the rotation curve of the gas, surface density maps, stellar surface density profiles and metallicity profiles. The most conservative choice is made to avoid spurious fits but to maintain consistency throughout the analysis.

early times, in simulated galaxies one might expect the spheroid mass to dominate over disk mass but this does not appear to be the case; although admittedly the spheroid-to-disk mass ratios of these galaxies are still not as low as typical bright spiral galaxies (Weinzirl et al. 2009). Also note from Fig. 3 that although the characteristic central peak in the rotation curve (associated with excessive concentrations of mass) is present, the decline of the rotation curve has more to do with the concentration of the disk. This issue affects all galaxies irrespective of environment and thus biases in the star formation history do not render comparison of the two environment samples unreliable.

The lack of a clear separation in the loose group and field populations in the spheroid-to-disk plot may point to the fact that the environments here are not sufficiently different to allow the galaxies to manifest different disk properties and that galaxies differ significantly only if they inhabit more extreme overdensities. It also reflects the dynamics of these groups. None of the galaxies have undergone much interaction with any other massive group member, having not passed pericenter with one another at $z=0$. This removes harassment by massive galaxies as a possible source of disruption and leaves only the possibility of complete mergers with smaller satellite galaxies than those shown in Fig. 2 as a possible explanation.

\subsection{Metallicity gradients}

We now examine the metallicity gradients of the galaxies for evidence of environmental influences. Metallicity gradients of 
Table 2. Galaxy properties of the simulated disks at $z \sim 0$ : scale length, metallicity gradient (for stars with age less than $100 \mathrm{Myr}$ ), cold gas mass-weighted metallicity average $\left(T<1.5 \times 10^{4} \mathrm{~K}\right)$, stellar mass (kinematically defined disk stars), cold gas mass, and magnitudes $(b$ and $r$ SDSS filters).

\begin{tabular}{lcccccccc}
\hline \hline Name & Environment & $\begin{array}{c}\text { Scale length } \\
(\mathrm{kpc})\end{array}$ & $\begin{array}{c}\mathrm{d}[Z] \mathrm{d} R_{\mathrm{GC}}^{-1} \\
(\mathrm{dex} \mathrm{kpc})\end{array}$ & Mean $[Z]$ & $\begin{array}{c}\text { Stellar disk mass } \\
\left(M_{\odot}\right)\end{array}$ & $\begin{array}{c}\text { Gas disk mass } \\
\left(M_{\odot}\right)\end{array}$ & $M_{\mathrm{b}}$ & $M_{\mathrm{r}}$ \\
\hline Castor & loose group & 3.88 & -0.034 & -0.194 & $7.19 \times 10^{10}$ & $3.32 \times 10^{10}$ & -21.67 & -22.30 \\
Pollux & loose group & 3.07 & -0.052 & -0.139 & $3.45 \times 10^{10}$ & $7.64 \times 10^{9}$ & -20.58 & -21.33 \\
Zeus & loose group & 1.76 & -0.044 & -0.159 & $1.03 \times 10^{10}$ & $6.25 \times 10^{9}$ & -19.70 & -20.52 \\
Tyndareus & loose group & 2.27 & -0.048 & -0.086 & $1.32 \times 10^{10}$ & $8.38 \times 10^{9}$ & -19.77 & -20.61 \\
Apollo & loose group & 2.86 & -0.057 & -0.269 & $6.30 \times 10^{10}$ & $2.25 \times 10^{10}$ & -21.22 & -22.00 \\
Artemis & loose group & 1.75 & -0.047 & -0.239 & $3.24 \times 10^{10}$ & $1.06 \times 10^{10}$ & -20.52 & -21.30 \\
Daphne & loose group & 2.64 & -0.060 & -0.139 & $2.14 \times 10^{10}$ & $1.40 \times 10^{10}$ & -20.20 & -20.92 \\
Leto & loose group & 1.56 & -0.057 & -0.207 & $1.19 \times 10^{10}$ & $7.51 \times 10^{9}$ & -20.32 & -21.01 \\
Luke & loose group & 5.19 & -0.035 & -0.164 & $6.61 \times 10^{10}$ & $4.58 \times 10^{10}$ & -21.30 & -22.05 \\
Leia & loose group & 3.94 & -0.019 & -0.130 & $3.03 \times 10^{10}$ & $1.22 \times 10^{10}$ & -20.22 & -21.06 \\
\hline Ben & field & 3.85 & -0.033 & -0.272 & $4.17 \times 10^{10}$ & $2.70 \times 10^{10}$ & -20.96 & -21.67 \\
Tethys & field & 2.77 & -0.050 & -0.231 & $5.12 \times 10^{10}$ & $1.52 \times 10^{10}$ & -21.24 & -21.97 \\
Krios & field & 2.50 & -0.051 & -0.204 & $3.99 \times 10^{10}$ & $1.19 \times 10^{10}$ & -20.61 & -21.43 \\
Atlas & field & 2.71 & -0.042 & -0.170 & $4.38 \times 10^{10}$ & $1.22 \times 10^{10}$ & -20.88 & -21.61 \\
Hyperion & field & 3.59 & -0.040 & -0.199 & $7.66 \times 10^{10}$ & $1.46 \times 10^{10}$ & -21.24 & -22.05 \\
Eos & field & 1.96 & -0.069 & -0.279 & $2.51 \times 10^{10}$ & $1.14 \times 10^{10}$ & -20.07 & -20.87 \\
Helios & field & 1.56 & -0.037 & -0.069 & $6.57 \times 10^{10}$ & $5.26 \times 10^{9}$ & -21.01 & -21.93 \\
Selene & field & 3.54 & -0.061 & -0.244 & $5.20 \times 10^{10}$ & $1.66 \times 10^{10}$ & -20.83 & -21.56 \\
Oceanus & field & 6.45 & -0.029 & -0.103 & $1.00 \times 10^{11}$ & $2.99 \times 10^{10}$ & -21.61 & -22.39 \\
\hline
\end{tabular}

Notes. Note that for the gas mass and metallicity determinations, spatial cuts are used to exclude satellites.

interacting and merged galaxies are known to be flatter (Ellison et al. 2008; Rupke et al. 2008; Kewley et al. 2010; Perez et al. 2011) and as such, abundance gradients provide a probe of the dynamical mixing. To make comparisons with observed HII gradients we select stars that are younger than 100 Myr from the kinematic decomposition and also employing the spatial constraints described in Sect. 2.4. Young stellar metallicity gradients are shown in Fig. 6 as a function of total galaxy mass. The gradients exhibited by the RaDES galaxies range from -0.07 to -0.02 dex $\mathrm{kpc}^{-1}$, consistent with observations by Zaritsky et al. (1994) of spiral galaxies in the field (-0.231 to $\left.0.021 \mathrm{dex} \mathrm{kpc}^{-1}\right)$. Gradients are also calculated for spiral galaxies in van Zee et al. (1998) spanning -0.07 to $-0.04 \mathrm{dex} \mathrm{kpc}^{-1}$ and Garnett et al. (1997) with a range of -0.083 to -0.020 dex $\mathrm{kpc}^{-1}$, the RaDES galaxies are remarkably close to these values.

Metallicity gradients are thought to be flatter for galaxies in denser environments and has been demonstrated to be true for close interacting binaries by Kewley et al. (2010) where HII region metallicity gradients are found between -0.040 to $-0.007 \mathrm{dex} \mathrm{kpc}^{-1}$. While there is no obvious distinction between the two samples presented here it is worth bearing in mind that the RaDES loose group galaxies are an order of magnitude more distant from each other than the galaxy pairs in Kewley et al. (2010). Furthermore the simulated gradients are not dramatically inconsistent with those measured for interacting binaries. We note that the simulated loose group galaxies do not have appreciably flatter young stellar gradients (with the exception of Leia), however they likewise do not have steeper gradients, which leaves the possibility that the statistical sizes of the samples here may be too small to probe such a slight effect.

Another feature of Fig. 6 is the trend for less massive galaxies to have steeper gradients. Leia is a notable outlier from this trend, having a particularly flat metallicity distribution, however when the gradient is calculated on stars of all ages the trend remains and Leia does not appear to be peculiar. This is counter to what might be expected since these galaxies have less massive dark matter haloes and therefore may be more easily perturbed and have flattened metallicity gradients. In Prantzos \& Boissier (2000), cosmologically motivated scaling relations are used to demonstrate that the metallicity gradients of spiral galaxies are steeper in less massive galaxies when expressed in dex $\mathrm{kpc}^{-1}$ but not so when expressed in dex $R_{\mathrm{d}}^{-1}$ where $R_{\mathrm{d}}$ is the disk scale length. This is a consequence of the shorter scale-length of the less massive disks arising from a steeper star formation rate profile which results in a greater metal production rate in the inner disk compared with the disk periphery. This behaviour is supported by observations (Garnett et al. 1997; van Zee et al. 1998) where it is shown that less luminous spiral galaxies have steeper gradients than brighter galaxies when the absolute gradient is measured. When observed gradients are normalised to the disk scale-length however no significant variation with luminosity is apparent. Another finding of Garnett et al. (1997) is that the dispersion of absolute gradients is larger for less luminous galaxies but when normalised the dispersion is consistent with brighter galaxies pointing to the existence of some degree of co-evolution of metallicity and density profiles. The scale length normalised metallicity gradients of young stars are shown in Fig. 7 and confirm that metallicity gradients likely have a common origin with the stellar density profile. This is explored in more depth in Pilkington et al. (2012) where clear links are made between the star formation profile and metallicity gradients. The absolute metallicity gradients of the RaDES galaxies are consistent with values found in literature (Garnett et al. 1997) however the normalised gradients are around an order of magnitude flatter suggesting that the measured density profiles are too steep, a feature that is consistent with the known issue of excess star formation at early times and peaked rotation curves in simulations (Navarro \& Benz 1991; Governato et al. 2004; Guo et al. 2010; Sawala et al. 2011). A result that is relevant to this work is that of Dutil \& Roy (1999). Here the authors find that HII metallicity gradients are flatter for earlier morphological types, but critically, that the trend is weaker when the gradients are normalised to some 
A\&A 547, A63 (2012)

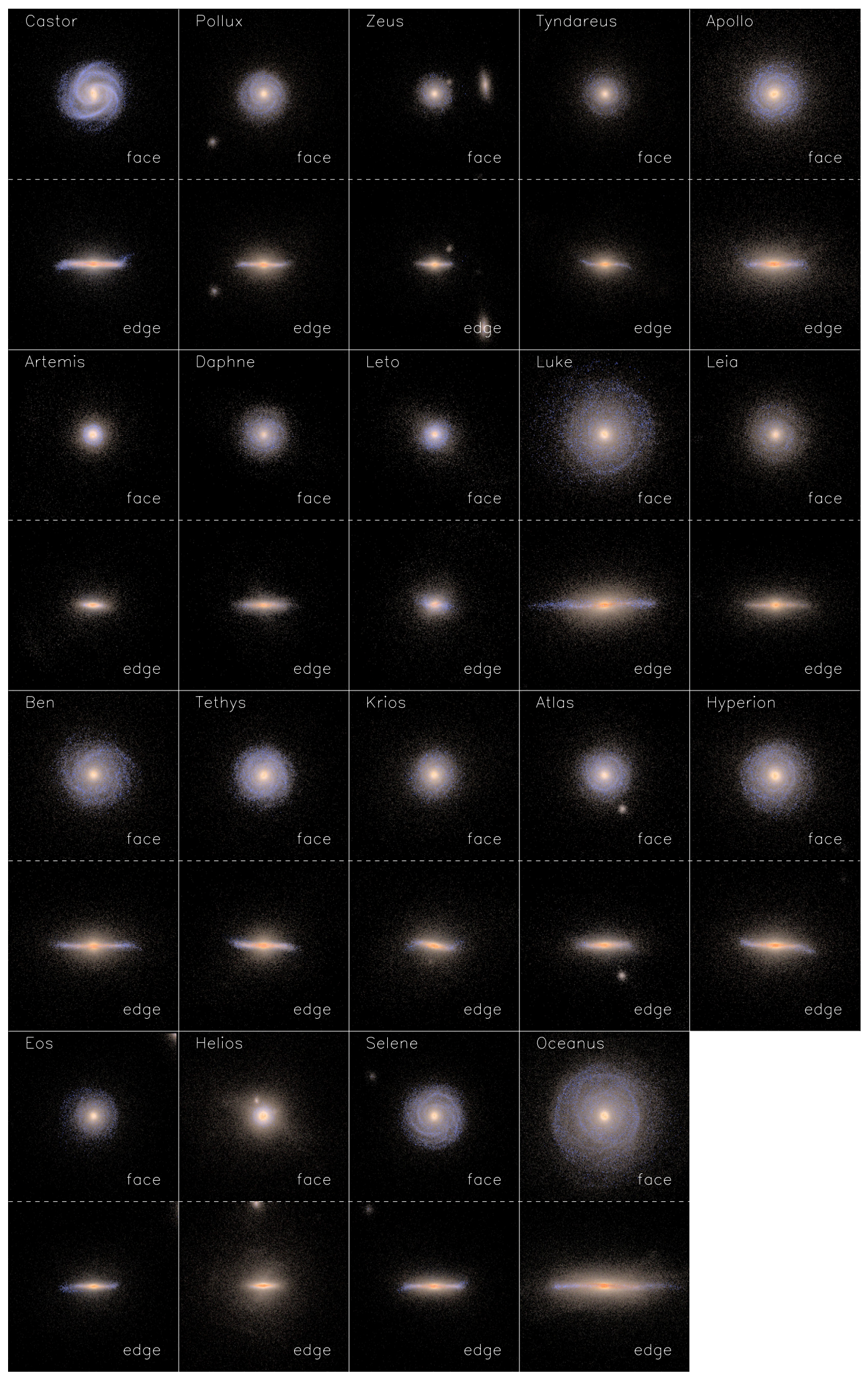

Fig. 4. Mock images of the galaxies. Face- and edge-on views are separated by dashed lines, images are created by combining SDSS $g, r$ and $i$ filters and are each $50 \times 50 \mathrm{kpc}^{2}$ in size. Simulation outputs at $z=0$ are used for all galaxies except Castor and Eos where the galaxies are shown at $z \simeq 0.03$. 
C. G. Few et al.: Properties of simulated Milky Way-mass galaxies

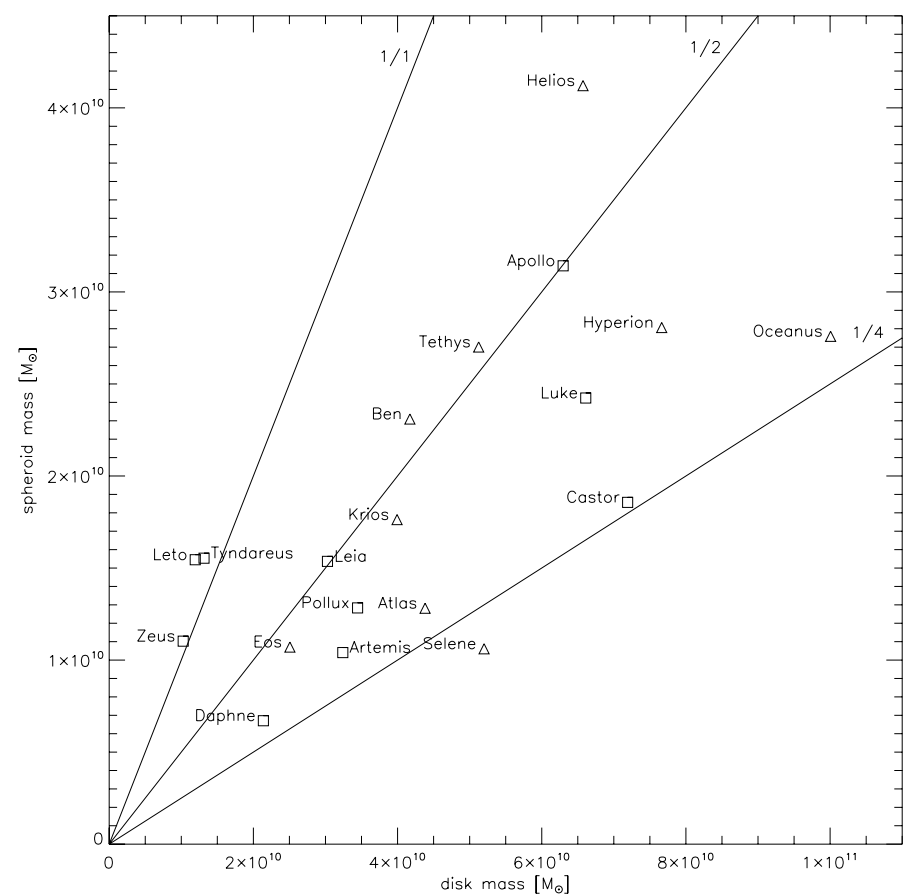

Fig. 5. Spheroid and disk masses as determined by kinematic decomposition of the $z \sim 0$ stellar phase. Squares and triangles indicate the loose group and field populations respectively. Radial lines have constant bulge-disk ratios.

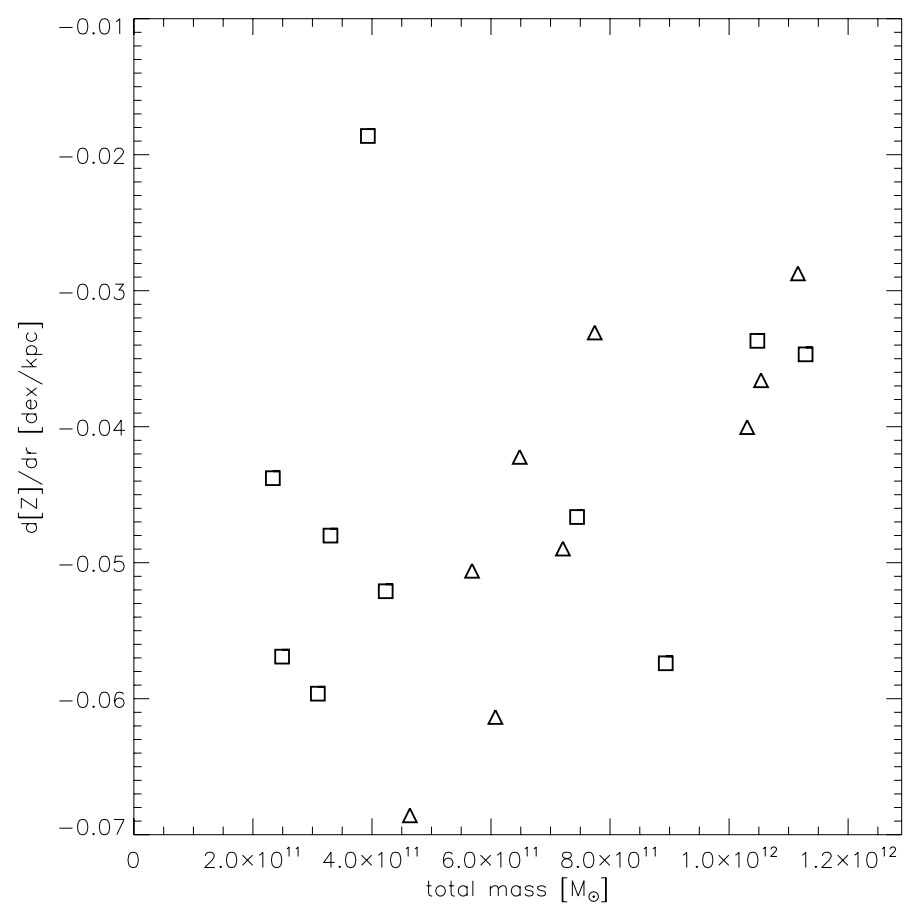

Fig. 6. Metallicity gradients of the disk stars at $z \sim 0$ plotted against the total mass of the galaxy halo, triangles represent field galaxies, squares are loose group galaxies. A trend towards shallower gradients with increasing mass is evident for both environment samples with a slight offset to flatter gradients for the loose group galaxies.

isophotal or effective radius. While we do not show the correlation between gradient and morphology (indeed no attempt is made to identify the classical morphology of these galaxies) it is clear that the metal gradient has some degree of co-evolution with the scale length.

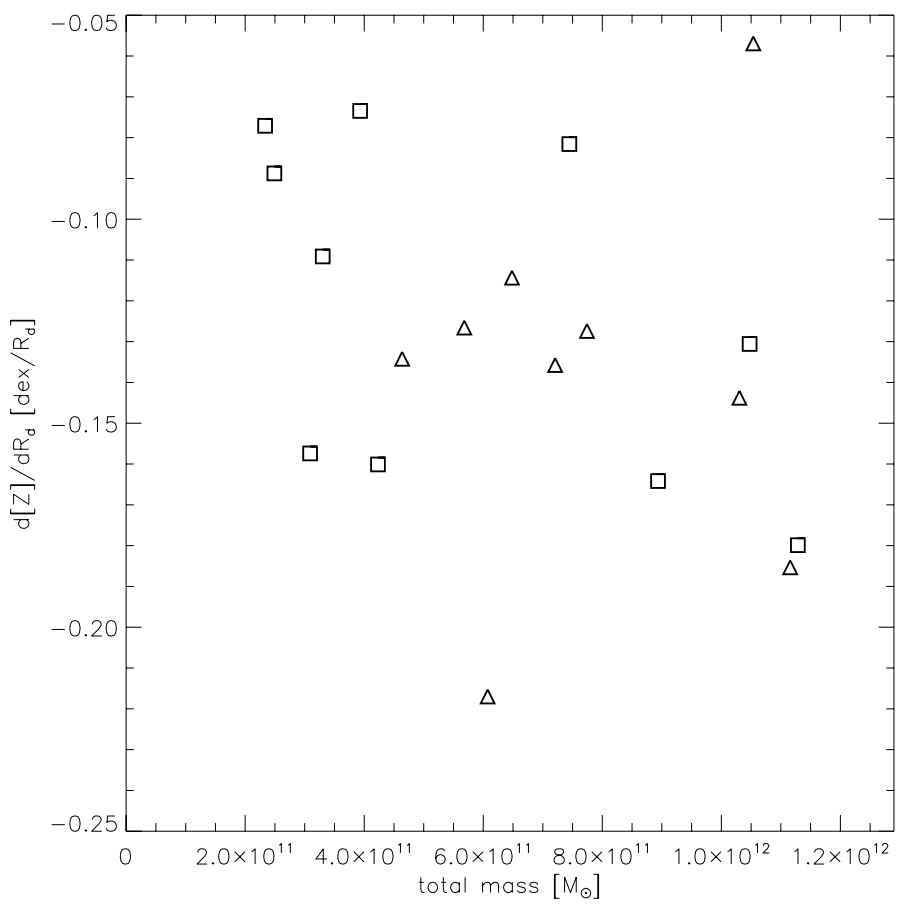

Fig. 7. Young stellar metallicity gradients at $z \sim 0$ normalised by stellar scale length plotted against the total galaxy mass, symbols are as in Fig. 6.

\subsection{Colour-magnitude diagram}

We use SUNRISE to produce seven different projections ranging from face-on to edge-on and display the values in a colour-magnitude diagram (Fig. 8) that overplots the observed (uncorrected) colour-magnitude diagram from SDSS data (Bailin \& Harris 2008). We represent the change in magnitude and colour as a function of projection angle for each galaxy in Fig. 8 with an arc that starts with a symbol denoting the faceon projection (symbol type follows paper conventions), the end of the arc denotes an edge on projection. Face-on magnitudes for each galaxy are given in Table 2. Almost all the galaxies populate the blue cloud with only Helios appearing within the red sequence. Much as expected, as the galaxies becomes more inclined they appear to dim and redden; many of the galaxies therefore have edge-on projections that stray into the dimmest end of the red sequence. There is a selection effect at work here as the galaxies are a priori chosen to be disk galaxies with ongoing star formation and that only Helios is particularly red is reassuring. The colour of the galaxies reflects that while the disk stars are too concentrated there is no critical over production of stars at high redshift which would bias the galaxies to the red regions of Fig. 8.

There is no apparent separation of the two samples once the obvious outlier of Helios has been discounted. There is some observational evidence that the colour distribution of late-type galaxies is only weakly dependent on environment and that it is more strongly influenced by the luminosity or mass through intrinsic evolution (Balogh et al. 2004b). The distribution in $r$-band magnitude is consistent with the mass of each galaxy and the colour of galaxies can be considered a probe of their star formation history (Fig. A.1). Taking Helios as an example, we see an initially prolonged star formation phase in comparison with other galaxies that have star formation rates that are less disparate throughout time. We examine the impulsive interactions of the disk with respect to kinematics in the next subsection. 


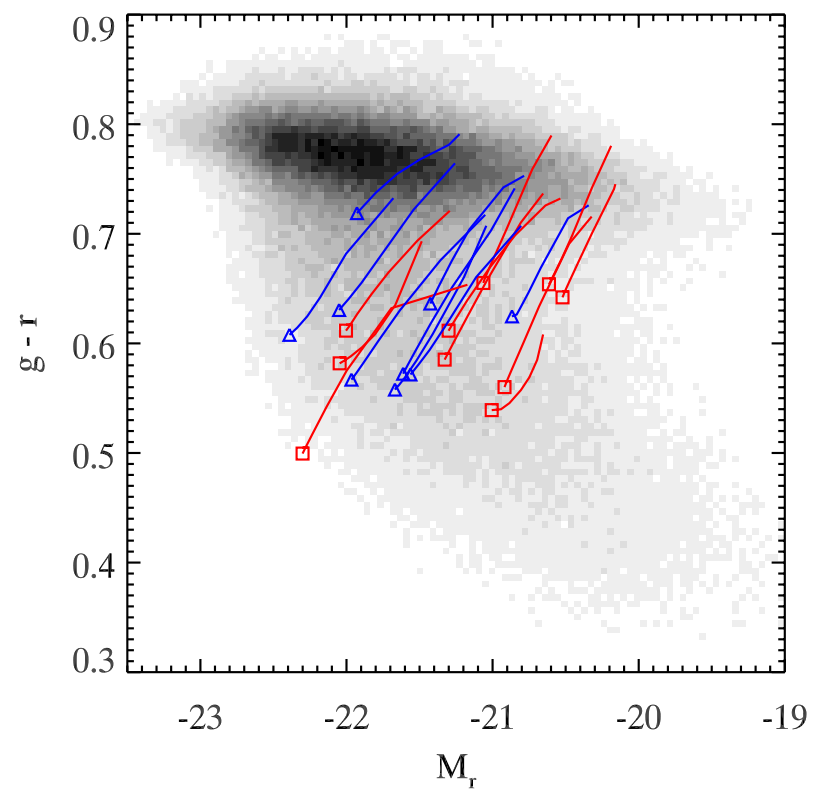

Fig. 8. Colour-magnitude diagram. The background data are SDSS galaxies with no inclination correction. Symbols follow the paper convention and denote the face-on values. The tails traces the change in orientation from face- to edge-on.

\subsection{Sigma-age relation}

We now move away from morphology and chemistry to examine kinematics and use the temporal behaviour of the stellar velocity dispersion $(\sigma)$ for this purpose. The velocity dispersion of a region analogous to the "solar neighbourhood" is used to quantify the influence that external interactions have on the kinematics of the disk. To remove the bias that arises from the velocity dispersion gradient as a function of disk radius we select stars from an annulus of thickness $2 \mathrm{kpc}$, centred on 3 times the disk scale length and a height of less than $3 \mathrm{kpc}$ above and below the equatorial plane. Figure 9 shows the velocity dispersion of stars, $\sigma$, as a function of their age at $z=0$. While many of the RaDES galaxies attain the observed velocity dispersion of the Milky Way (10-20 $\mathrm{km} \mathrm{s}^{-1}$ found by Soubiran \& Girard 2005; Soubiran et al. 2008; Holmberg et al. 2007) when the youngest stars are considered, the older populations have far greater dispersions than observed. The $\sigma$-age dispersion relations shown in Fig. 9 exhibit a greater increase in dispersion as a function of age and the appearance of more discrete steps than are apparent in observations. Greater velocity dispersions are to be expected in simulations as less than ten resolution elements are found in the would-be thin-disk (if such a structure were resolvable). The high early velocity dispersions found even in field galaxies with the fewest mergers (e.g. Krios or Selene) may indicate that these galaxies experience excess kinematic heating from mergers. Another possibility is that very early in the galaxies formation cold flows (rather than discrete mergers) are responsible for forming the majority of stars. As it is well known that cosmologically simulated galaxies form too many stars at early times (see Table 1 for the stellar mass fractions) it is possible that the high velocity dispersion of old stars is simply reflecting this shortcoming. We note however that maps of the gas distribution at high redshift clearly show discrete gaseous objects merging and no evidence is found for cold streams after $z=2$.

The $\sigma$-age relations do not at first display any obvious distinction between field and loose group environments, so a more rigorous analysis is called for. We have attempted to quantify the difference between a "stepped" and a smooth $\sigma$-age relation by looking for spikes in the age-derivative of $\sigma(a g e)$. If the age-derivative of $\sigma($ age $)$ exhibits spikes above some significance level (the step-threshold) it will betray the existence of steps in the velocity dispersion. Galaxies that have steps with magnitude exceeding a threshold (the step-threshold) that is a factor of $\beta$ greater than the average of the age-derivative of $\sigma$ are defined as having a "stepped profile". The value of $\beta$ is chosen in the range 4-7 as values that are too low will not discriminate small variations in the age-derivative from larger steps and a value that is too large will classify all steps as being normal. This test is repeated for different spatial selections in each galaxy (annuli with $2-4 \times R_{\mathrm{d}}$ in radius with a width of $\pm 2-4 \mathrm{kpc}$ including all stars within $3 \mathrm{kpc}$ of the disk plane) to avoid bias that may arise from sampling a spiral arm and for different values of beta in the range 4-7. In each case the field sample has approximately half the number of galaxies with step features than does the loose group sample. An example is shown in Fig. 9 for $\beta=5$ (for stars in an annulus of thickness $6 \mathrm{kpc}$ centred on $2 \times R_{\mathrm{d}}$ and a height of $3 \mathrm{kpc}$ above and below the equatorial plane) in which seven out of ten loose group galaxies have stepped profiles while only three of the nine field galaxies do. The binning in age usually places at least several hundred stars in each age bin making the uncertainty in the velocity dispersion (and propagated uncertainty in the discrete age-derivative) very small, these are plotted as 2 sigma error bars in Fig. 9. In the extreme case of taking $\beta=8$ none of the field galaxies have detected steps while four of the loose group galaxies do.

While each choice of annulus and $\beta$ results in the loose group sample having a greater fraction of galaxies with stepped profiles than the field sample, the number of galaxies studied here is not enough for this result to be conclusive and a more complete sample would be required to make it so. Nevertheless we now discuss the implications of the trend if it is real. The physical mechanism shaping the $\sigma$-age relation can be simply thought of as stars being heated to greater dispersion by all mergers subsequent to their formation and to an extent that depends on the severity of the merger (Villalobos \& Helmi 2008; Di Matteo et al. 2011). A series of gentle mergers therefore results in a smoother decline in dispersion towards younger stars (Kazantzidis et al. 2008) while a large and disruptive merger excites all stars formed previously to a plateau that gives a more step-like appearance to the $\sigma$-age relation (Brook et al. 2004). This contrasts with the conclusions of House et al. (2011) where stars are found to form with a velocity dispersion and retain it as a signature of the gas state at that time, however in either scenario the analysis performed in this work is a valid measure of the effect of disk disruption on stellar dynamics. The results shown in this work tentatively suggest that in spite of a superficially similar major-merger history, there may be some differences in the mergers experienced by the galaxy disk or the interaction histories. Firstly galaxy disk major mergers could impact the galaxies differentially depending on the gas fraction (Cox et al. 2006; Hopkins et al. 2009; Lotz et al. 2010), the orbital configuration (Barnes 2002; Robertson et al. 2006) and the large-scale tidal field (Martig \& Bournaud 2008). Secondly, the number of minor mergers or interactions with orbiting satellites could have an impact in shaping the $\sigma$-age relation (Quinn et al. 1993; Abadi et al. 2003; Bournaud et al. 2005). Third, the interaction of the galaxy with the intragroup medium may have an impact as found by Bekki \& Couch (2011) wherein the authors find that repetitive harassment in groups can lead to star formation bursts and disk heating. 
C. G. Few et al.: Properties of simulated Milky Way-mass galaxies

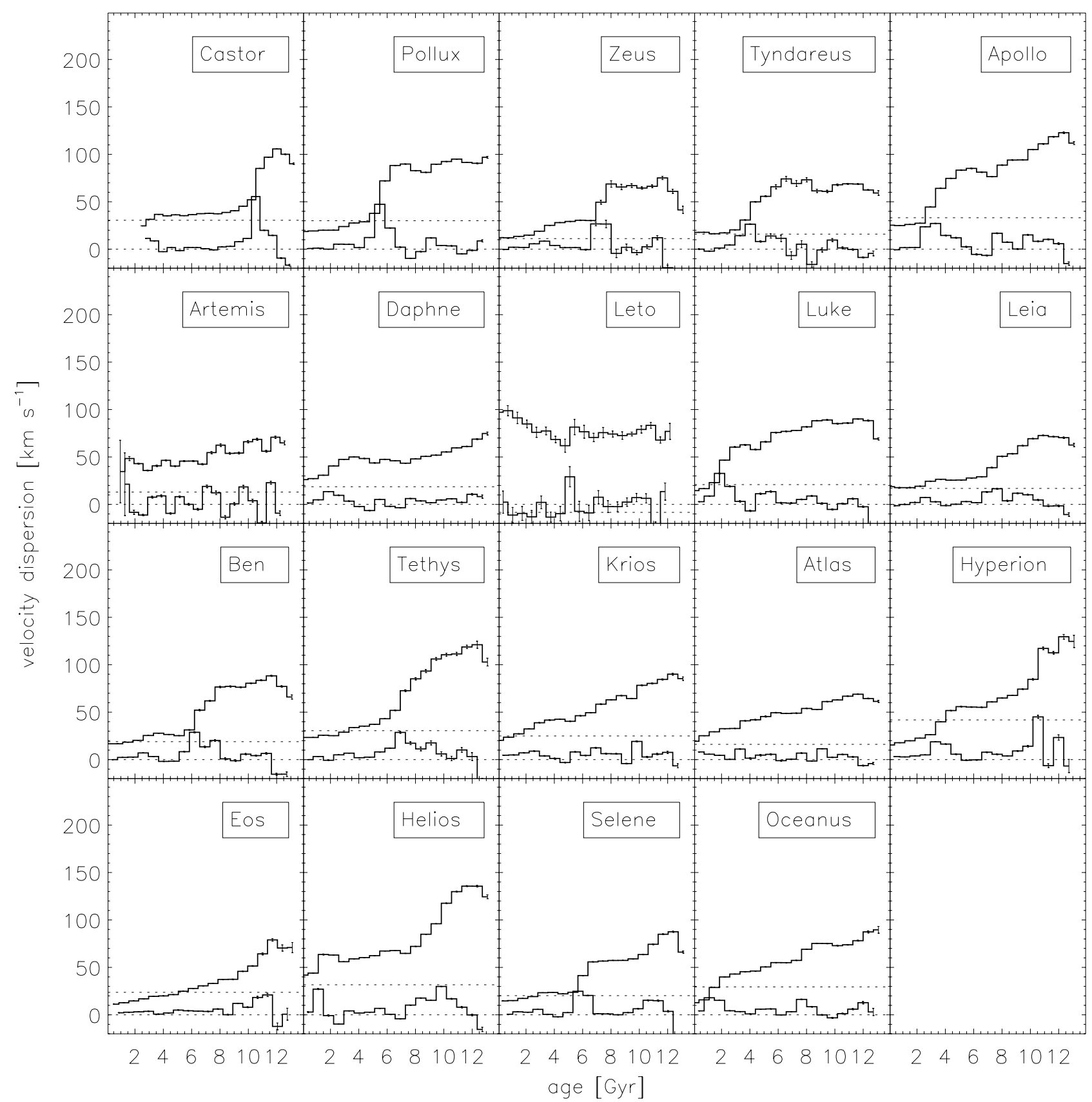

Fig. 9. Present day $(z=0$ in most cases, see Sect. 2.3) stellar velocity dispersion as a function of age for an annulus of thickness $6 \mathrm{kpc}$ centred on $2 \times R_{\mathrm{d}}$ and a height of $3 \mathrm{kpc}$ above and below the equatorial plane. The lower line is the age-derivative of this function, $\mathrm{d} \sigma / \mathrm{d} a g e$. Horizontal dotted lines define the zero point and a step-threshold that is $5 \times\langle\mathrm{d} \sigma / \mathrm{d} a g e\rangle$.

\section{Conclusions}

We present a suite of cosmological simulations with the intention of comparing field galaxies with galaxies in Local Group environments. The galaxies are taken from cosmological simulations where a zoom method is used to allow sub-kpc resolution while simultaneously accounting for large scale structure formation. A kinematic decomposition has been performed to separate disk stars from spheroid stars and we have analysed the morphology of the galaxies. While all galaxies studied here have larger stellar mass fractions than observations dictate they should, this issue is uniform and does not affect comparisons between the two environments. We have also examined the metallicity gradients finding trends with mass but a very weak or non-existent correlation with environment. Finally the stellar velocity dispersion is studied and evidence of a dependence on environment is found in the signature of impulsive heating in group galaxies. The results of this work are pertinent to the comparison of simulated field galaxies with observations of the Milky Way. The conclusions of this work are summarised here:

1. No distinction between loose group and field galaxies is seen when considering the spheroid-to-disk ratio although examination of galaxies with greater spheroidal components shows that they all have interactions that disturb their disk rather than forming from kinematically hotter gas. However this is far from conclusive as there are only four galaxies with significantly higher spheroidal components of the total 19 . 
2. Metallicity gradients of loose group galaxies are very similar to those of field galaxies with the same disk mass, a result that is still consistent with observations of strongly interacting galaxies (Kewley et al. 2010) though non conclusive evidence is seen that loose group galaxies should have significantly flatter gradients compared with their counterparts in the field. The absolute gradients are consistent with observations (Zaritsky et al. 1994; Garnett et al. 1997; van Zee et al. 1998 ) yet when normalised by disk scale-length gradients are an order of magnitude flatter than observed suggesting that density profiles are too concentrated. Observations also show that more massive spiral galaxies have flatter gradients, this has previously been matched by semi-analytical models (Prantzos \& Boissier 2000) using scaling relations but the trend has now also been shown to exist for our numerically simulated galaxies. We also find a link between metallicity gradients and stellar density gradients that suggests that galaxies in the mass range studied here have similar metal gradients when expressed in $\operatorname{dex} / R_{\mathrm{d}}$ and that variance in this value may be attributable to radial migration or disruptive mergers.

3. Examination of the age-velocity dispersion relation reveals that as expected the velocity dispersion of old stars in the simulated galaxies is greater than observed for the Milky Way disk. Loose group galaxies exhibit more stepped relations that suggest mergers/harassment do have a greater impact on the loose group galaxies than field galaxies. This is at odds with the apparent similarities in the major merger frequency of the two samples and suggests that the major merger history of dark matter haloes may not be an accurate probe of the galaxy disk merger history. We note however that the relatively small sample size means that it is not conclusive whether or not this is a real effect depending on environment or simply because the loose group galaxies happen to have more turbulent formation histories independent of environment; certainly the individual formation history of each galaxy will impact on its velocity dispersion evolution and this would be consistent with the other findings of this work.

The main conclusion to come from this work is that in such sparse environments where the galaxies are not directly interacting galaxies exhibit different properties depending on individual merger histories and infall rates but that loose groups environments are only very weakly different to the field. It has been suggested for cluster galaxies that it is likely that galaxies are shaped more by direct mergers and their own secular behaviour rather than the large-scale environment that impacts the aforementioned only indirectly (McGee et al. 2008). Structures further than $\sim 1 \mathrm{Mpc}$ distant have little influence for galaxies with masses presented here.

The method used to define mergers in this work may be insufficient to link mergers to the signatures of the impact they have on the disk properties and a future study to follow this should develop a larger suite using only dark matter simulations to quantify the satellite distribution and minor merger rates with a greater statistical significance. Simulations at higher resolution should also be employed to determine conclusively if any systematic difference in metallicity gradients exists. We finish by stating that at the resolutions considered here simulated galaxies may be safely compared with Milky Way properties whether they inhabit loose group or field environments, however attention must be given to the aggregated merger properties, mass and internal structure for such comparisons to be meaningful.
Acknowledgements. The authors would like to acknowledge Romain Teyssier for both access to RAMSES and for helpful discussions regarding its use. We are grateful to referee Sebastien Peirani for the many suggestions that have greatly improved this manuscript. C.G.F. acknowledges the support of STFC through its Ph.D. Studentship programme (ST/F007701/1). B.K.G., C.B.B., S.C., L.M.D., acknowledge the support of the UK's Science \& Technology Facilities Council (ST/F002432/1 \& ST/H00260X/1). B.K.G. acknowledges the generous visitor support provided by Saint Mary's and Monash Universities. We also acknowledge the UK's National Cosmology Supercomputer (COSMOS), and the University of Central Lancashire's High Performance Computing Facility. S.C. acknowledges support from the BINGO Project (ANR-08-BLAN-0316-01). L.M.D. acknowledges support from the Lyon Institute of Origins under grant ANR-10-LABX-66.

\section{References}

Abadi, M. G., Navarro, J. F., Steinmetz, M., \& Eke, V. R. 2003, ApJ, 597, 21 Aubert, D., Pichon, C., \& Colombi, S. 2004, MNRAS, 352, 376

Bailin, J., \& Harris, W. E. 2008, ApJ, 681, 225

Balogh, M., Eke, V., Miller, C., et al. 2004a, MNRAS, 348, 1355

Balogh, M. L., Baldry, I. K., Nichol, R., et al. 2004b, ApJ, 615, L101

Bamford, S. P., Nichol, R. C., Baldry, I. K., et al. 2009, MNRAS, 393, 1324

Barnes, J. E. 2002, MNRAS, 333, 481

Barnes, J. E., \& Hernquist, L. 1996, ApJ, 471, 115

Barton, E. J., Geller, M. J., \& Kenyon, S. J. 2000, ApJ, 530, 660

Bekki, K., \& Couch, W. J. 2011, MNRAS, 415, 1783

Bournaud, F., Jog, C. J., \& Combes, F. 2005, A\&A, 437, 69

Brook, C. B., Kawata, D., Gibson, B. K., \& Freeman, K. C. 2004, ApJ, 612, 894 Brooks, A. M., Solomon, A. R., Governato, F., et al. 2011, ApJ, 728, 51

Cooper, M. C., Tremonti, C. A., Newman, J. A., \& Zabludoff, A. I. 2008, MNRAS, 390, 245

Cox, T. J., Dutta, S. N., Di Matteo, T., et al. 2006, ApJ, 650, 791

Di Matteo, P., Lehnert, M. D., Qu, Y., \& van Driel, W. 2011, A\&A, 525, L3

Donzelli, C. J., \& Pastoriza, M. G. 2000, AJ, 120, 189

Dressler, A. 1980, ApJ, 236, 351

Dressler, A., Oemler, Jr., A., Couch, W. J., et al. 1997, ApJ, 490, 577

Dubois, Y., \& Teyssier, R. 2008, A\&A, 477, 79

Dutil, Y., \& Roy, J.-R. 1999, ApJ, 516, 62

Ellison, S. L., Patton, D. R., Simard, L., \& McConnachie, A. W. 2008, AJ, 135, 1877

Ellison, S. L., Simard, L., Cowan, N. B., et al. 2009, MNRAS, 396, 1257

Fabbiano, G., Baldi, A., King, A. R., et al. 2004, ApJ, 605, L21

Garnett, D. R., Shields, G. A., Skillman, E. D., Sagan, S. P., \& Dufour, R. J 1997, ApJ, 489, 63

Giuricin, G., Limboz Tektunali, F., Monaco, P., Mardirossian, F., \& Mezzetti, M. 1995, ApJ, 450, 41

Gómez, P. L., Nichol, R. C., Miller, C. J., et al. 2003, ApJ, 584, 210

Gottloeber, S., Hoffman, Y., \& Yepes, G. 2010 [arXiv: 1005.2687]

Governato, F., Mayer, L., Wadsley, J., et al. 2004, ApJ, 607, 688

Governato, F., Willman, B., Mayer, L., et al. 2007, MNRAS, 374, 1479

Guedes, J., Callegari, S., Madau, P., \& Mayer, L. 2011, ApJ, 742, 76

Guo, Q., White, S., Li, C., \& Boylan-Kolchin, M. 2010, MNRAS, 404, 111

Haardt, F., \& Madau, P. 1996, ApJ, 461, 20

Hernquist, L. 1989, Nature, 340, 687

Holmberg, J., Nordström, B., \& Andersen, J. 2007, A\&A, 475, 519

Hopkins, P. F., Somerville, R. S., Cox, T. J., et al. 2009, MNRAS, 397, 802

House, E. L., Brook, C. B., Gibson, B. K., et al. 2011, MNRAS, 415, 2652

Jonsson, P. 2006, MNRAS, 372, 2

Kazantzidis, S., Bullock, J. S., Zentner, A. R., Kravtsov, A. V., \& Moustakas, L. A. 2008, ApJ, 688, 254

Kewley, L. J., Geller, M. J., \& Barton, E. J. 2006, AJ, 131, 2004

Kewley, L. J., Rupke, D., Zahid, H. J., Geller, M. J., \& Barton, E. J. 2010, ApJ, 721, L48

Kobayashi, C., \& Nakasato, N. 2011, ApJ, 729, 16

Lambas, D. G., Tissera, P. B., Alonso, M. S., \& Coldwell, G. 2003, MNRAS, 346,1189

Leauthaud, A., Tinker, J., Bundy, K., et al. 2012, ApJ, 744, 159

Leitherer, C., Schaerer, D., Goldader, J. D., et al. 1999, ApJS, 123, 3

Lewis, I., Balogh, M., De Propris, R., et al. 2002, MNRAS, 334, 673

Libeskind, N. I., Knebe, A., Hoffman, Y., et al. 2011, MNRAS, 411, 1525

Lilly, S. J., Le Fevre, O., Hammer, F., \& Crampton, D. 1996, ApJ, 460, L1

Lotz, J. M., Jonsson, P., Cox, T. J., \& Primack, J. R. 2010, MNRAS, 404, 590

Mandelbaum, R., Seljak, U., Kauffmann, G., Hirata, C. M., \& Brinkmann, J. 2006, MNRAS, 368, 715

Márquez, I., Masegosa, J., Moles, M., et al. 2002, A\&A, 393, 389

Martig, M., \& Bournaud, F. 2008, MNRAS, 385, L38

Martínez, H. J., Coenda, V., \& Muriel, H. 2008, MNRAS, 391, 585 
C. G. Few et al.: Properties of simulated Milky Way-mass galaxies

Martinez-Vaquero, L. A., Yepes, G., Hoffman, Y., Gottlöber, S., \& Sivan, M. 2009, MNRAS, 397, 2070

McGee, S. L., Balogh, M. L., Henderson, R. D. E., et al. 2008, MNRAS, 387, 1605

Michel-Dansac, L., Lambas, D. G., Alonso, M. S., \& Tissera, P. 2008, MNRAS, 386, L82

Montuori, M., Di Matteo, P., Lehnert, M. D., Combes, F., \& Semelin, B. 2010, A\&A, 518, A56

Moore, B., Katz, N., Lake, G., Dressler, A., \& Oemler, A. 1996, Nature, 379, 613

Moster, B. P., Somerville, R. S., Maulbetsch, C., et al. 2010, ApJ, 710, 903

Navarro, J. F., \& Benz, W. 1991, ApJ, 380, 320

Nikolic, B., Cullen, H., \& Alexander, P. 2004, MNRAS, 355, 874

Peirani, S. 2010, MNRAS, 407, 1487

Peirani, S., Jung, I., Silk, J., \& Pichon, C. 2012, MNRAS, accepted [arXiv: 1205 . 4694]

Perez, J., Michel-Dansac, L., \& Tissera, P. B. 2011, MNRAS, 417, 580

Pilkington, K., Few, C. G., Gibson, B. K., et al. 2012, A\&A, 540, A56

Prantzos, N., \& Boissier, S. 2000, MNRAS, 313, 338

Quinn, P. J., Hernquist, L., \& Fullagar, D. P. 1993, ApJ, 403, 74

Rahimi, A., Kawata, D., Brook, C. B., \& Gibson, B. K. 2010, MNRAS, 401, 1826

Robertson, B., Yoshida, N., Springel, V., \& Hernquist, L. 2004, ApJ, 606, 32

Robertson, B., Bullock, J. S., Cox, T. J., et al. 2006, ApJ, 645, 986

Rupke, D. S. N., Veilleux, S., \& Baker, A. J. 2008, ApJ, 674, 172

Rupke, D. S. N., Kewley, L. J., \& Chien, L.-H. 2010, ApJ, 723, 1255
Sánchez-Blázquez, P., Courty, S., Gibson, B. K., \& Brook, C. B. 2009, MNRAS, 398,591

Sawala, T., Guo, Q., Scannapieco, C., Jenkins, A., \& White, S. 2011, MNRAS, 413, 659

Scannapieco, C., Tissera, P. B., White, S. D. M., \& Springel, V. 2005, MNRAS, 364,552

Scannapieco, C., White, S. D. M., Springel, V., \& Tissera, P. B. 2009, MNRAS, 396, 696

Snaith, O. N., Gibson, B. K., Brook, C. B., et al. 2011, MNRAS, 415, 2798

Sol Alonso, M., Michel-Dansac, L., \& Lambas, D. G. 2010, A\&A, 514, A57

Soubiran, C., \& Girard, P. 2005, A\&A, 438, 139

Soubiran, C., Bienaymé, O., Mishenina, T. V., \& Kovtyukh, V. V. 2008, A\&A, 480, 91

Stinson, G. S., Bailin, J., Couchman, H., et al. 2010, MNRAS, 408, 812

Teyssier, R. 2002, A\&A, 385, 337

Tweed, D., Devriendt, J., Blaizot, J., Colombi, S., \& Slyz, A. 2009, A\&A, 506, 647

van Zee, L., Salzer, J. J., Haynes, M. P., O’Donoghue, A. A., \& Balonek, T. J. 1998, AJ, 116, 2805

Villalobos, Á., \& Helmi, A. 2008, MNRAS, 391, 1806

Weinmann, S. M., van den Bosch, F. C., Yang, X., \& Mo, H. J. 2006, MNRAS, 366,2

Weinzirl, T., Jogee, S., Khochfar, S., Burkert, A., \& Kormendy, J. 2009, ApJ, 696, 411

Zaritsky, D., Kennicutt, Jr., R. C., \& Huchra, J. P. 1994, ApJ, 420, 87

Pages 14 to 16 are available in the electronic edition of the journal at http://www . aanda. org 


\section{Appendix A: Additional galaxy properties}

Here we describe some properties of the galaxies that are not directly discussed in the main paper body but are still useful as supplementary material. We begin with some general comments on particular features of each galaxy that are of interest.

- Castor is the only galaxy in the sample to exhibit a bar, perhaps reflecting the greater resolution or the more isotropic nature of its group (compared with, for example, the filamentary structure of the Apollo group). It also presents the clearest example of spiral structure of the entire sample. The spiral structure presents challenges when quantifying the stellar scale length as the arms present a bump in the density profile. The young stars present in the arms mean that this is even more pronounced when measuring the brightness profile. Castor also has the most pronounced (and abrupt) disk warp, initially this was believed to be evidence of poor resolution at the disk edge but investigation has revealed no particularly favoured alignment of the disk warps in this sample. Analysis of Castor has been conducted on a snapshot slightly before $z=0$ due to the irregular shape induced by a late merger in this snapshot.

- Artemis is unusual in that it has a relatively massive dark matter halo $\left(7.45 \times 10^{11} M_{\odot}\right)$, reasonably low spheroid-to-disk fraction $(0.32)$ and flat metallicity profile $(-0.0068 \mathrm{dex} / \mathrm{kpc})$, yet its disk scale length is only $1.87 \mathrm{kpc}$ and is truncated at a radius of $7 \mathrm{kpc}$. There exists a gaseous polar disk (not dense enough to form stars) and yet the vertical velocity dispersion changes very little as a function of age. This suggests that the last major merger experienced by Artemis left star-forming gas with a similar velocity dispersion to the older stars, an effect not seen in the other galaxies.

- Leto is the least massive galaxy within the Apollo group and the most spheroid dominated of the galaxies. This spheroid is not composed of older stars as in the other galaxies, there is a significant fraction of the spheroid stars that are young. This is the result of a low star formation rate at early times compounded by a recent, disruptive event that is evident in the velocity dispersion-age relation.

- Eos undergoes a merger at late times that leaves it with an extremely irregular morphology at the last time step, analysis of this galaxy is performed on a snapshot prior to this event.

- Helios is the most early type galaxy of all. Despite its great mass it is the reddest galaxy and has young stars with around twice the vertical velocity dispersion of much of the rest of the sample and a prolonged early star formation episode, this contrasts with the lack of an identifiable late merger to result in such a morphology.

- Selene has few mergers in its history and is one of the most quiescent galaxies, forming the largest disk fraction of all the galaxies and presenting definite spiral structure.

- Oceanus has the greatest stellar mass in the sample (though it is among others with comparable halo masses) and has a rotating gaseous disk that extends as far as $40 \mathrm{kpc}$ from the centre. This disk is dense enough to form stars and hence this galaxy has an extremely long scale length $(6.63 \mathrm{kpc})$ and one of the flattest metallicity gradients.

One of the key ways of understanding the formation of galaxies is by examining the star formation history of the different components. The distribution of star formation in time tells us a great deal about how the different components of a galaxy form. In the course of this work the signature of mergers were found to be identifiable in the bursts of star formation seen in Fig. A.1. These bursts can in some cases be associated with steps in the velocity dispersion described in Sect. 3.4, however we find that the magnitude of the star formation bursts is a poor indicator of the strength of the kinematic disturbance induced by the merger from which they both originate. The dichotomy of the star formation burst and the kinematic effects of mergers is yet more evidence that the signature of a merger depends on the gas fraction or phase space configuration of the merging bodies. Note the restrained recent star formation of the disrupted galaxy Artemis compared with the more disk dominated Apollo or Oceanus.

For the analysis of the simulated disk galaxies presented in this work to be as uniform as possible we performed a kinematic decomposition to examine the disk stars with minimal contamination from halo and bulge stars. The decomposition employed follows the Abadi et al. (2003) method of using the angular momentum of stars compared with the angular momentum expected for rotating stars. The distribution of the relative angular momentum is shown in Fig. A.2 with blue and red lines highlighting the distribution on disk and spheroid stars respectively. 
C. G. Few et al.: Properties of simulated Milky Way-mass galaxies

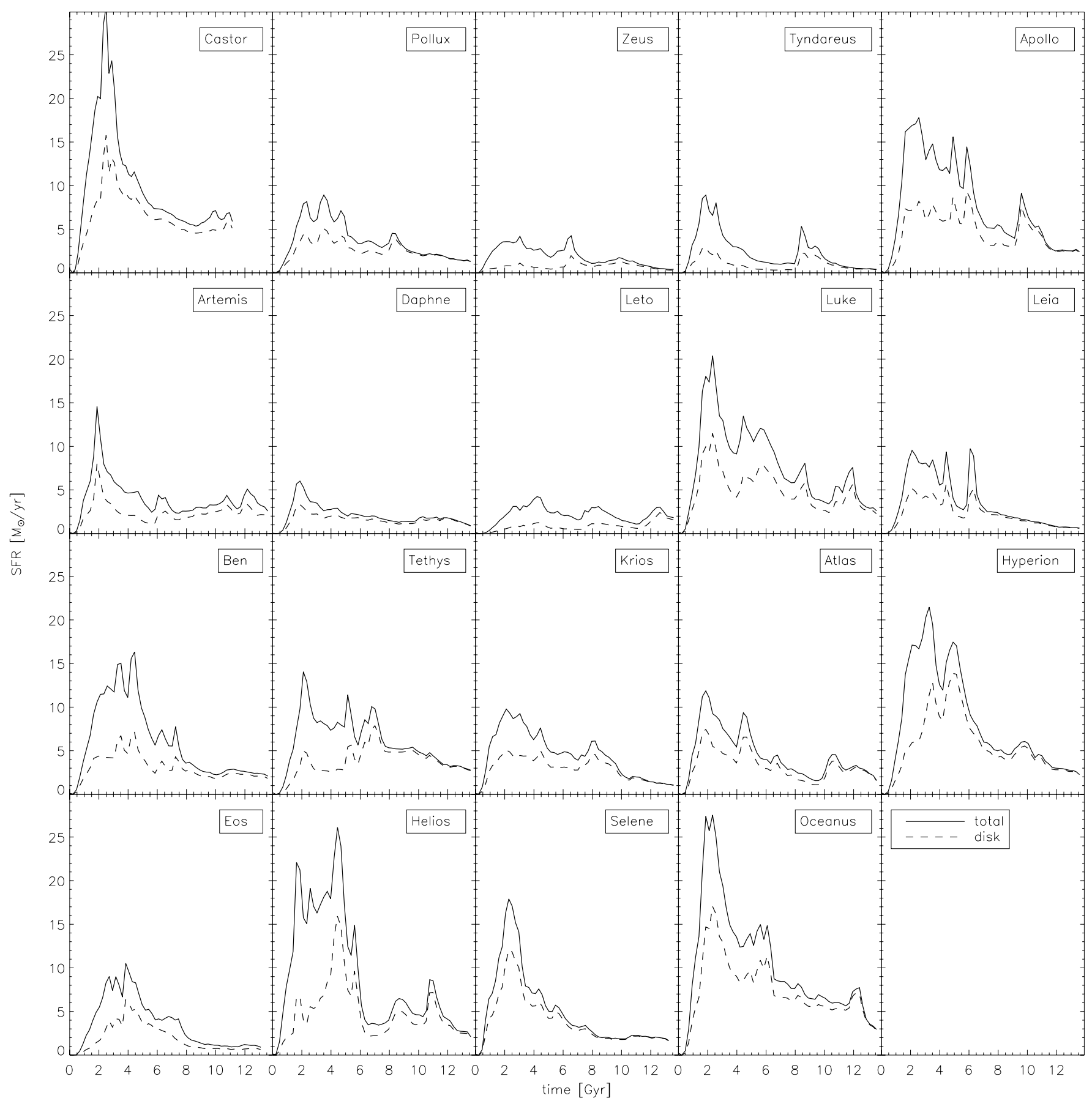

Fig. A.1. Star formation histories of the sample galaxies. The star formation rate of all stars within the virial radius at $z=0$ are shown by the solid line, the dashed line is all stars tagged as disk stars at $z=0$. 
A\&A 547, A63 (2012)

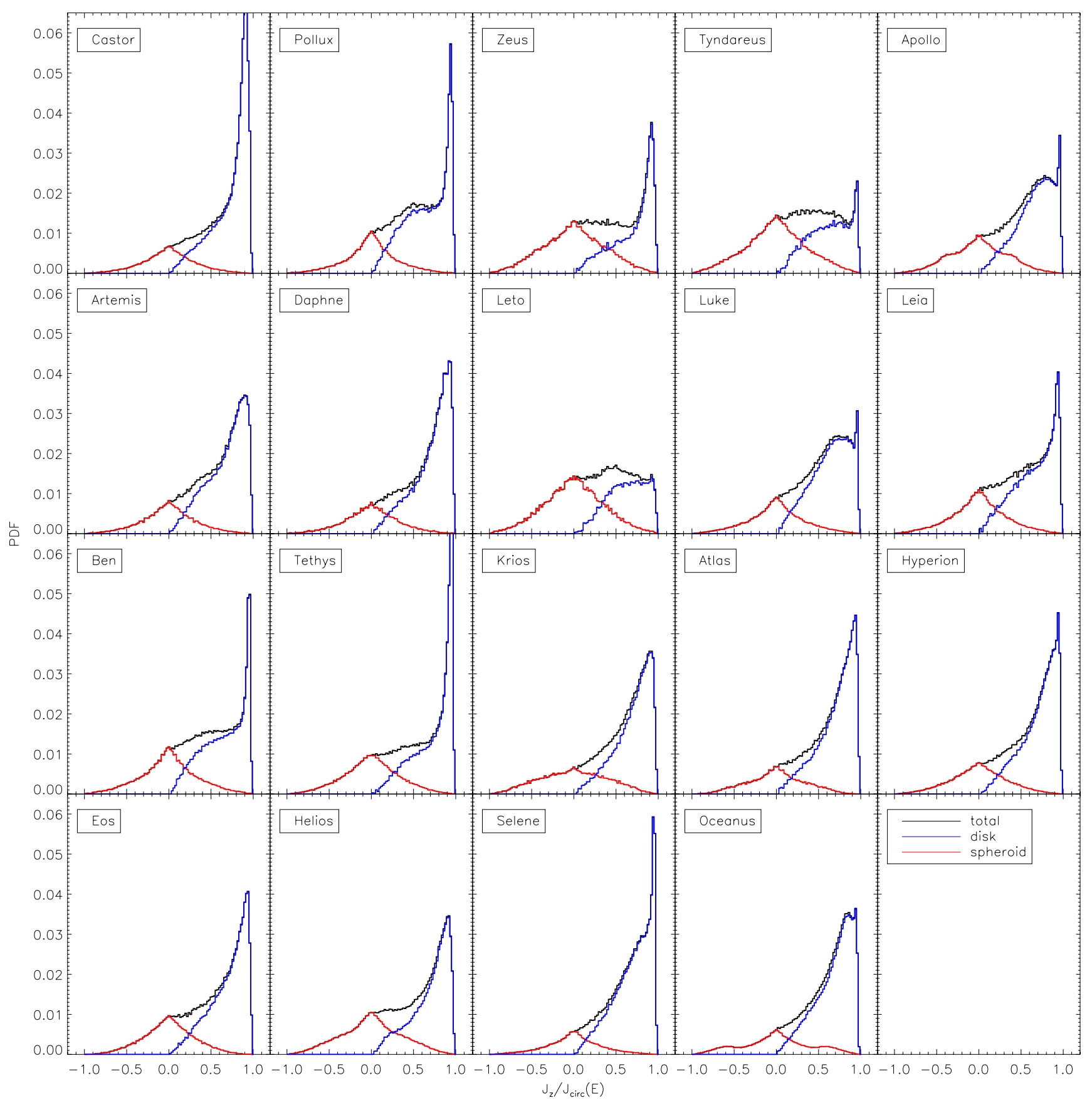

Fig. A.2. $J_{z} / J_{\text {circ }}$ distribution of stars within the virial radius (black). Blue and red lines shows the distribution of the disk and spheroid components respectively. Note the existence of a third intermediate component included in the distribution that is associated with the disk in some of the galaxies. 